\title{
Direct Synthesis of Co-doped Graphene on Dielectric Substrates Using Solid Carbon Sources
}

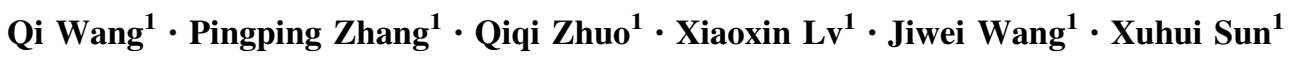

Received: 25 May 2015/Accepted: 30 June 2015/Published online: 16 July 2015

(C) The Author(s) 2015. This article is published with open access at Springerlink.com

\begin{abstract}
Direct synthesis of high-quality doped graphene on dielectric substrates without transfer is highly desired for simplified device processing in electronic applications. However, graphene synthesis directly on substrates suitable for device applications, though highly demanded, remains unattainable and challenging. Here, a simple and transfer-free synthesis of high-quality doped graphene on the dielectric substrate has been developed using a thin $\mathrm{Cu}$ layer as the top catalyst and polycyclic aromatic hydrocarbons as both carbon precursors and doping sources. N-doped and N, F-co-doped graphene have been achieved using TPB and $\mathrm{F}_{16} \mathrm{CuPc}$ as solid carbon sources, respectively. The growth conditions were systematically optimized and the as-grown doped graphene were well characterized. The growth strategy provides a controllable transfer-free route for high-quality doped graphene synthesis, which will facilitate the practical applications of graphene.
\end{abstract}

Keywords Graphene $\cdot$ Solid carbon sources $\cdot$ Transfer-free $\cdot$ Doping and co-doping

\section{Introduction}

Graphene, a one-atom-thick layer of carbon with $s p^{2}$ hybrid orbital bonding and two-dimensional structure material, has attracted intense research interests due to its extraordinary physical and chemical characteristics, such as good mechanical strength [1], high carrier mobility [2], excellent electrical conductivity [3], superior thermal conductivity [4], and high transmittance [5]. However, the nature of pristine graphene with zero band gap brings some difficulties for its application in the electronic device field [6].

Electronic supplementary material The online version of this article (doi:10.1007/s40820-015-0052-6) contains supplementary material, which is available to authorized users.

Xuhui Sun

xhsun@suda.edu.cn

1 Jiangsu Key Laboratory for Carbon-Based Functional Materials and Devices, Institute of Functional Nano \& Soft Materials, Soochow University, Suzhou 215123, Jiangsu, People's Republic of China
Doping of graphene with other heteroatoms (e.g., nitrogen, boron, phosphorus, halogen, etc.) is the most practicable, convenient, and efficient approach to modulate the band structure and properties of graphene [7] and further extend more useful applications in electronics and electrochemical cells [8-13].

Among all the approaches to synthesize doped graphene, chemical vapor deposition (CVD) is the most popular method to obtain high-quality doped graphene in large scale by introducing copper or nickel foil as the catalyst [3, 14, 15] and independent doping source (e.g., $\mathrm{NH}_{3}$ as $\mathrm{N}$ doping source) [16, 17]. Recently, carbon sources containing dopant element have been used to directly grow doped graphene by CVD method, avoiding the post-doping treatment or using dopant gases in the growth process. For example, Tour et al. demonstrated a new approach that large area, high-quality $\mathrm{N}$-doped graphene with controllable thickness can be grown from different solid carbon sources such as polymer films or small molecules, deposited on a metal catalyst substrate at $800{ }^{\circ} \mathrm{C}$ [18]. Liu et al. developed a self-assembly approach that allows the synthesis of single-layer and highly nitrogen-doped graphene 
domain arrays by self-organization of pyridine molecules on the $\mathrm{Cu}$ surface [9]. However, the graphene film obtained by these methods generally requires physical transfer onto the desired substrates for subsequent device processing [19, 20], which could introduce the defects and contaminations into the graphene film.

Recently, we have developed a new transfer-free approach capable of synthesizing graphene directly on dielectric substrates using polycyclic aromatic hydrocarbons (PAHs) as carbon sources [21]. Significantly, N doping and patterning of graphene can be readily and concurrently achieved by this growth method. In this paper, we systematically investigate the factors that affect the growth quality of the doped graphene and optimized the growth conditions for high-quality doped graphene. Furthermore, we demonstrate that N, F-co-doped graphene can be synthesized using only $1,2,3,4,8,9,10,11,15,16,17$, 18, 22, 23, 24, 25-Hexadecafluorophthalocyanine Copper(II) $\left(\mathrm{F}_{16} \mathrm{CuPc}\right)$ as solid carbon source and both $\mathrm{N}$ and $\mathrm{F}$ doping sources.

\section{Experimental Section}

The schematic of growth process of doped graphene directly on $\mathrm{SiO}_{2}$-layered $\mathrm{Si}\left(\mathrm{SiO}_{2} / \mathrm{Si}\right)$ without transfer is shown in Fig. 1. First, the $\mathrm{SiO}_{2} / \mathrm{Si}\left(\mathrm{SiO}_{2}: 300 \mathrm{~nm}\right.$ thick) substrate was ultrasonically cleaned by acetone, ethanol, and deionized water for $15 \mathrm{~min}$, respectively. Then PAHs with planar structure (TPB or $\mathrm{F}_{16} \mathrm{CuPc}$ ) were evaporated on the substrate as solid carbon sources by thermal evaporation system (Organic Evaporation Coating Machine ZZBU500SA), followed by the $\mathrm{Cu}$ film layer deposition on the surface of PAHs as catalyst by electron-beam evaporation system (Kurt J. Lesker, PVD750). After annealing in a tube furnace under $\mathrm{Ar}$ gas flow at $\sim 1.8 \times 10^{2} \mathrm{~Pa}$, doped graphene was synthesized between the $\mathrm{Cu}$ layer and the substrate. At last, $\mathrm{Cu}$ layer was etched away by Marble's reagent $\left(\mathrm{CuSO}_{4}: \mathrm{HCl}: \mathrm{H}_{2} \mathrm{O}=10 \mathrm{~g}: 50 \mathrm{~mL}: 50 \mathrm{~mL}\right)$, then doped graphene was obtained directly on $\mathrm{SiO}_{2}$ substrate without any transfer process.

The morphology of doped graphene was characterized by scanning electron microscopy (SEM) (FEI Quanta 200F). Raman spectra were recorded at room temperature using a Jobin-Yvon HR800 Raman microscope with laser excitation at $514 \mathrm{~nm}$. Optical images were obtained using Fluorescence optical microscope (DM4000M). The HRTEM images were taken by transmission electron microscope (TEM) (Tecnai G2 F20). The surface state and electron structure of the samples were studied by X-ray photoelectron spectroscopy (XPS) measurement (Kratos AXIS UltraDLD ultrahigh vacuum (UHV) surface analysis system), using $\mathrm{Al} K \alpha \mathrm{X}$-rays $(1486 \mathrm{eV})$ as the excitation source. The optical transmittance spectrum and sheet

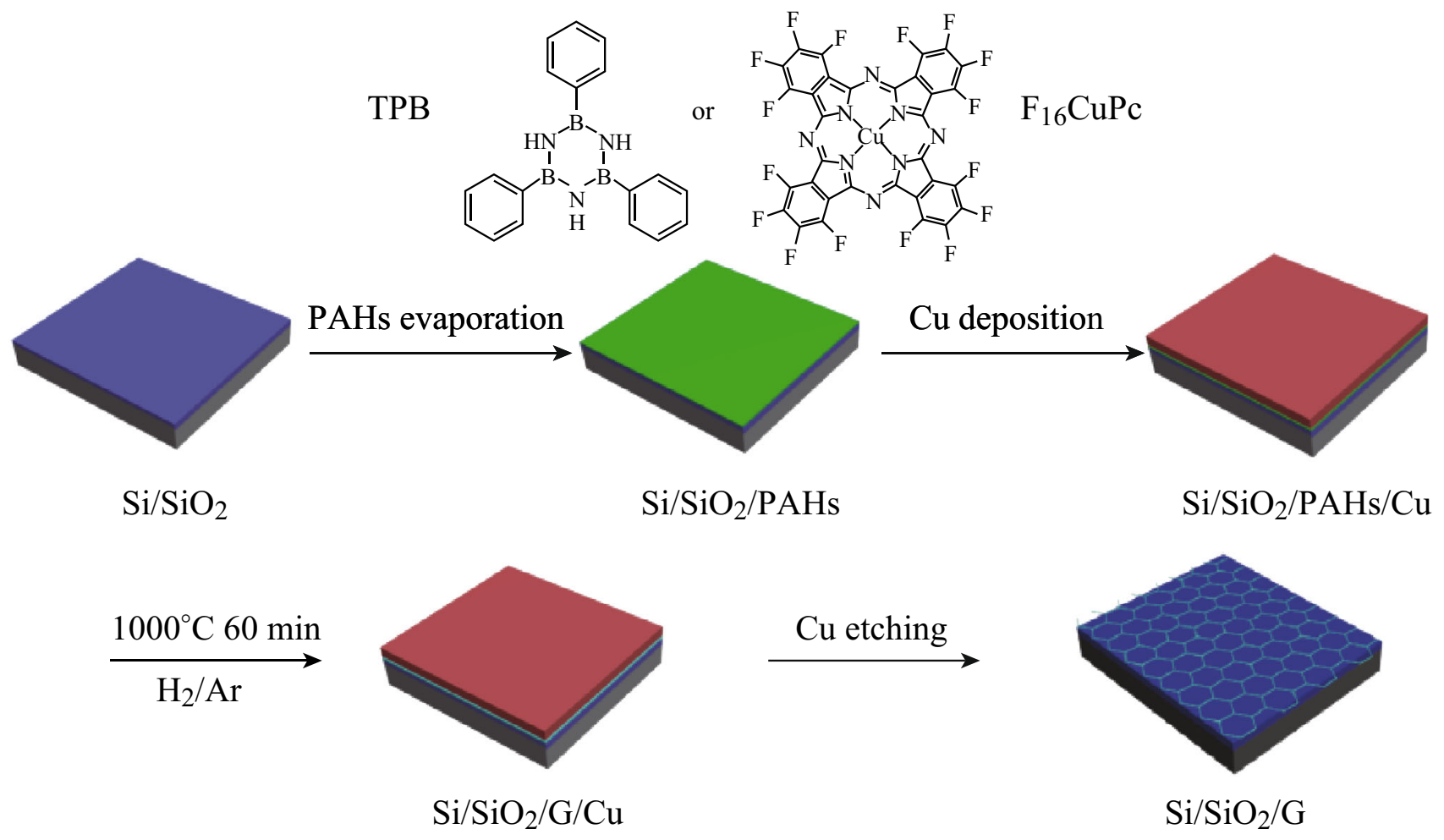

Fig. 1 Schematic representation of the doped graphene synthesis process 
resistance of doped graphene are measured by ultravioletvisible-near-infrared spectrophotometer (LAMBDA 750) and four-point probe system (ST-2258A), respectively.

\section{Result and Discussion}

Carbon source is an important factor in graphene synthesis. We found that planar configuration of PAHs might provide a hexagonal honeycomb skeleton structure for the graphene growth and the growth mechanism from PAHs maybe involves surface-mediated nucleation process of dehydrogenated PAHs catalyzed by $\mathrm{Cu}$ rather than segregation or precipitation process of small carbon species that decomposed from the precursors. Therefore, planar PAHs that contain heteroatoms (e.g., nitrogen, boron, fluorine) were chosen as solid carbon sources for doped graphene growth in our work. In addition to the specific structure of solid carbon sources, there are some other key factors to control the quality of doped graphene, such as the thickness of solid carbon sources, the thickness of $\mathrm{Cu}$ film layer, annealing time, annealing temperature, etc. Hence, in order to achieve high-quality doped graphene, optimal conditions for doped graphene growth had been investigated by rationalizing the above factors. 2, 4, 6-triphenylborazine (TPB) with planar configuration was selected as the solid carbon source to evaluate the growth conditions of the doped graphene.

The thickness effect of TPB on the quality of graphene was investigated firstly. The Raman spectra shown in Fig. S1a reveal that the optimum thickness of TPB layer is $5 \mathrm{~nm}$. When the thickness of TPB is less than $5 \mathrm{~nm}$, the carbon source cannot form continuous film on the substrate, which could result in the formation of discontinuous graphene. While the thickness of TPB is greater than $5 \mathrm{~nm}$, the excessive amount of carbon source leads to multilayer graphene or amorphous carbon formation due to the extremely low solubility of $\mathrm{C}$ in $\mathrm{Cu}$. Different annealing temperatures were also investigated for the growth of TPB-derived doped graphene. In general, the growth temperature, in conventional CVD method, required to synthesize good-quality graphene is $1000-1050{ }^{\circ} \mathrm{C}$. Figure S1b shows the Raman spectra of graphene synthesized at different growth temperatures, suggesting that graphene can be obtained above $950{ }^{\circ} \mathrm{C}$. Annealing temperature below $650{ }^{\circ} \mathrm{C}$ results in the deposition of amorphous carbon, as characterized by the broad $\mathrm{D}$ and $\mathrm{G}$ bands and a very weak $2 \mathrm{D}$ band shown in Fig. S1b. When the annealing temperature was increased to $1050{ }^{\circ} \mathrm{C}$, the obtained graphene layer also has a larger $\mathrm{D}$ band than that grown at $1000{ }^{\circ} \mathrm{C}$ in the Raman spectra. It has probably arisen from the partial evaporation of thin $\mathrm{Cu}$ film at $1050{ }^{\circ} \mathrm{C}$.
Subsequently, different annealing times were studied. As shown in Fig. S1c, higher quality doped graphene with lower $I_{\mathrm{D}} / I_{\mathrm{G}}$ and higher $I_{2 \mathrm{D}} / I_{\mathrm{G}}$ ratio can be achieved when the annealing time is $60 \mathrm{~min}$. The effect of $\mathrm{Cu}$ film thickness on doped graphene growth was investigated as well. When $\mathrm{Cu}$ film thickness is above $100 \mathrm{~nm}$, the graphene film can be obtained. However, when the thickness of the $\mathrm{Cu}$ film was decreased below $100 \mathrm{~nm}$, most of the $\mathrm{Cu}$ was evaporated during the annealing process at $1000{ }^{\circ} \mathrm{C}$ and resulted in discontinuous doped graphene. In addition, the graphene formed on the top surface of $\mathrm{Cu}$ was observed when a thin $\mathrm{Cu}$ layer was used. When the thickness of the $\mathrm{Cu}$ film was increased to $1000 \mathrm{~nm}$, relatively high-quality doped graphene was obtained indicated by the G/2D ratio $(\sim 0.3), \mathrm{D} / \mathrm{G}$ ratio $(\sim 1.3)$, and FWHM of the 2D $\left(\sim 42 \mathrm{~cm}^{-1}\right)$ band in Fig. S1d. Thus the optimal growth conditions for the doped graphene growth from TPB were set at $5 \mathrm{~nm}$ TPB as carbon source, $1000 \mathrm{~nm} \mathrm{Cu}$ film on the top surface, and annealing temperature of $1000{ }^{\circ} \mathrm{C}$ for $60 \mathrm{~min}$.

Figure 2a shows the optical image of the doped graphene grown on $\mathrm{SiO}_{2} / \mathrm{Si}$ substrate at the optimal condition using TPB as the carbon source. The continuous film with almost no contrast indicates that the graphene is distributed uniformly on the dielectric substrate. The corresponding Raman spectrum in Fig. 2b shows a weak D band, revealing that the graphene film is almost defect free and the weak D band may have arisen from the doping effect. The G/2D ratio is $\sim 0.25$ and the $2 \mathrm{D}$ peak is sharp and symmetric, indicating that the obtained graphene is monolayer $[22,23]$. The monolayer graphene is also confirmed by AFM measurement as shown in Fig. S2. A small D' band beside $G$ band confirms that doped graphene has been obtained. Figure S3 shows micro-Raman mapping for the $2 \mathrm{D}$ graphene peak, further indicating that graphene film is distributed uniformly on the substrate. Figure $2 \mathrm{c}$ shows the high-resolution XPS scan of N 1s centered at $400.7 \mathrm{eV}$, further confirming that $\mathrm{N}$-doped graphene was obtained under this optimal condition. All the results demonstrate that planar configuration of PAHs precursor containing dopant elements promotes the formation of doped graphene. The atomic concentration of $\mathrm{N}$ in TPB-derived doped graphene is about $1.74 \%$ from the data of XPS survey scan. No B 1s peak was observed for this sample, which is probably owing to the difficulty of $\mathrm{B}-\mathrm{C}$ bonding formation in graphene film at the present condition.

In order to achieve co-doping in graphene by this method, $\mathrm{F}_{16} \mathrm{CuPc}$ of $5 \mathrm{~nm}$ was used as the carbon source to prepare N, F-co-doped graphene on the $\mathrm{SiO}_{2} / \mathrm{Si}$ substrate at $1000{ }^{\circ} \mathrm{C}$ for $60 \mathrm{~min} . \mathrm{F}_{16} \mathrm{CuPc}$ is also a PAH compound with a planar structure. The Raman spectrum of the product (Fig. 3a) shows a large D peak and a small D' peak, which may be induced by $\mathrm{N}$ and $\mathrm{F}$ doping atoms. The G/2D ratio 

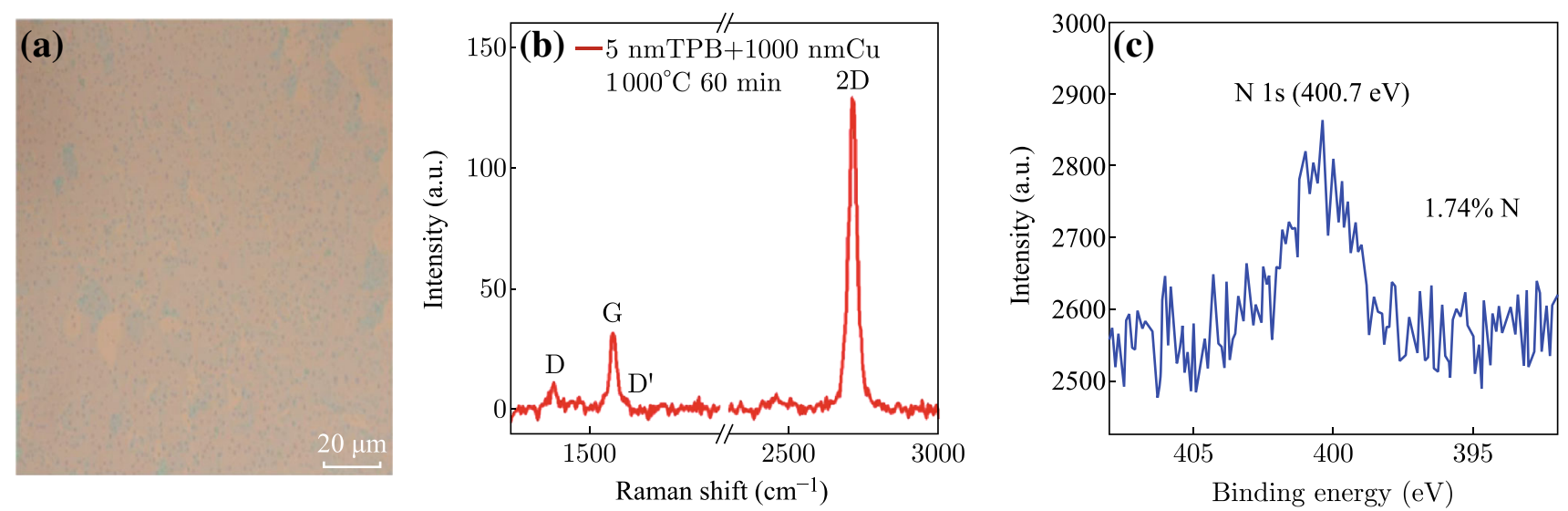

Fig. 2 a Optical image, b Raman spectrum, and $\mathbf{c}$ the high-resolution XPS scan of N 1s of doped graphene grown from $5 \mathrm{~nm} \mathrm{TPB,} 1000 \mathrm{~nm} \mathrm{Cu}$, annealing at $1000{ }^{\circ} \mathrm{C}$ for $60 \mathrm{~min}$
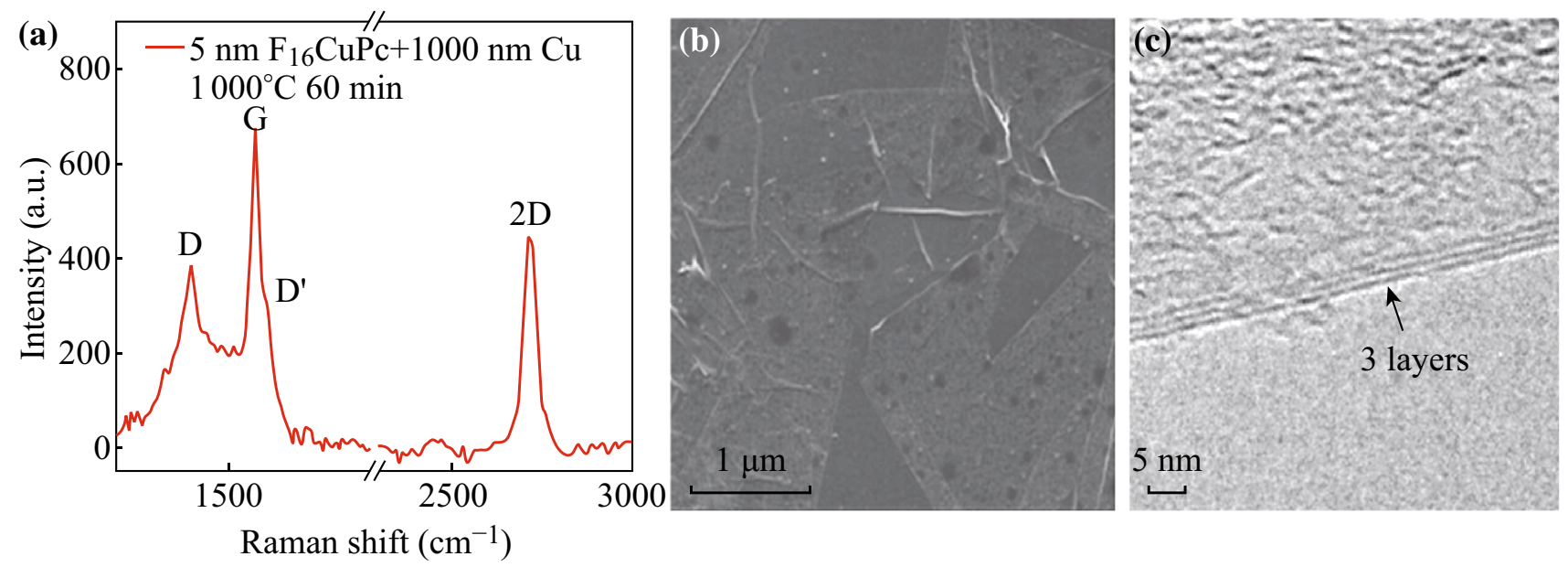

Fig. 3 a Raman spectrum, b SEM image, and c HR-TEM image of $\mathrm{F}_{16} \mathrm{CuPc}$-derived N, F-co-doped graphene

is $\sim 1.5$ and the $2 \mathrm{D}$ peak is lower and broader than that of the single layer graphene, indicating that the obtained doped graphene film is of 3-4 layers. Figure 3b shows SEM image of $\mathrm{F}_{16} \mathrm{CuPc}$-derived doped graphene. It can be found that thin graphene film is homogeneously distributed on the substrate. Moreover, it can be clearly distinguished from the HR-TEM image shown in Fig. 3c that the doped graphene film is of three layers, which is consistent with Raman analysis.

XPS investigation further verifies that $\mathrm{N}$ and $\mathrm{F}$ codoping has been achieved in the graphene. XPS spectra of N, F-co-doped graphene are shown in Fig. 4. Figure 4a shows the full XPS spectrum of $\mathrm{F}_{16} \mathrm{CuPc}$-derived doped graphene on the $\mathrm{SiO}_{2} / \mathrm{Si}$ substrate. There is no signal of $\mathrm{Cu}$, indicating the clear removal of $\mathrm{Cu}$ after etching. Both the nitrogen- and fluorine-related peaks are obviously found in the survey scan, which confirms the successful co-doping of $\mathrm{N}$ and $\mathrm{F}$ in the graphene film. The atomic concentration of $\mathrm{N}$ and $\mathrm{F}$ for $\mathrm{F}_{16} \mathrm{CuPc}$-derived doped graphene is about 2.98 and $0.66 \%$, respectively. The characteristic XPS C 1s core-level spectrum (Fig. 4b) is assigned as $s p^{2}$ carbon $(284.4 \mathrm{eV})$, confirming the graphitic structure of the as-grown graphene grains. The shoulder around 285.5 and $286.6 \mathrm{eV}$ can be assigned to the $\mathrm{C}-\mathrm{N}$ and $\mathrm{C}-\mathrm{F}$ bonding, respectively. Figure $4 \mathrm{c}$ shows the high-resolution XPS scan of $\mathrm{N} 1 \mathrm{~s}$, suggesting two types of N-C bonding: "graphitic" $\mathrm{N}$ centered at $401.1 \mathrm{eV}$ and "pyridine" $\mathrm{N}$ centered at $399.2 \mathrm{eV}$ [7, 9, 18]. The ratio of two types $\mathrm{N}$ indicates that they are mainly bonded to three adjacent carbons, suggesting that the $\mathrm{N}$ atoms are uniformly bound to the graphene structure. The high-resolution XPS scan of F 1s shows a single symmetric peak centered at $689.1 \mathrm{eV}$ in Fig. 4d, which is assigned to C-F covalent bond [24].

Figure 5 shows the result of optical transmittance measurement for the N, F-co-doped graphene directly grown on quartz in the same condition as using $\mathrm{SiO}_{2} / \mathrm{Si}$ substrate, exhibiting a high optical transmittance of 

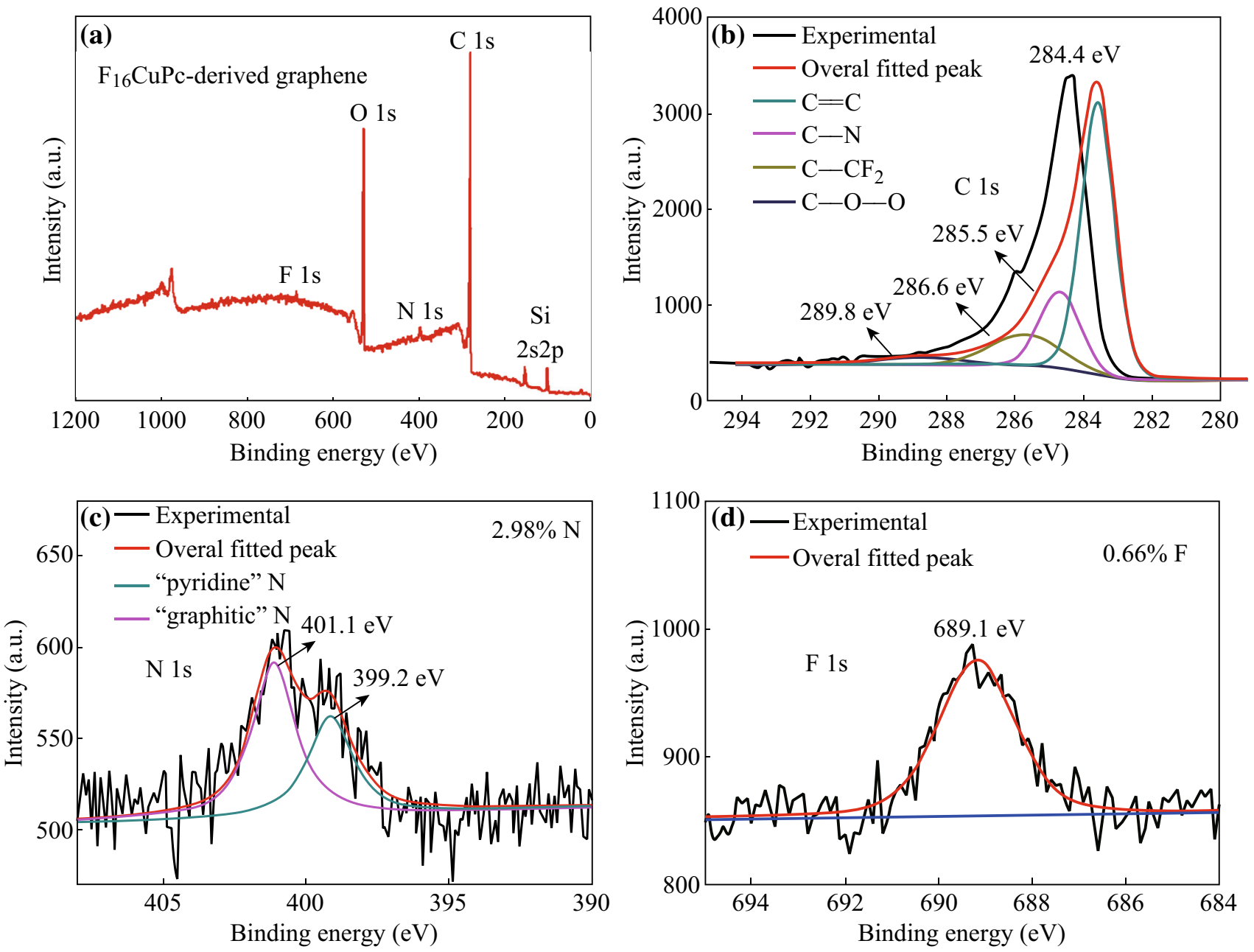

Fig. 4 XPS spectroscopic analysis of $\mathrm{F}_{16} \mathrm{CuPc}$-derived N, F-co-doped graphene. a Full XPS spectrum, b C 1s, c N 1s, and d F 1s

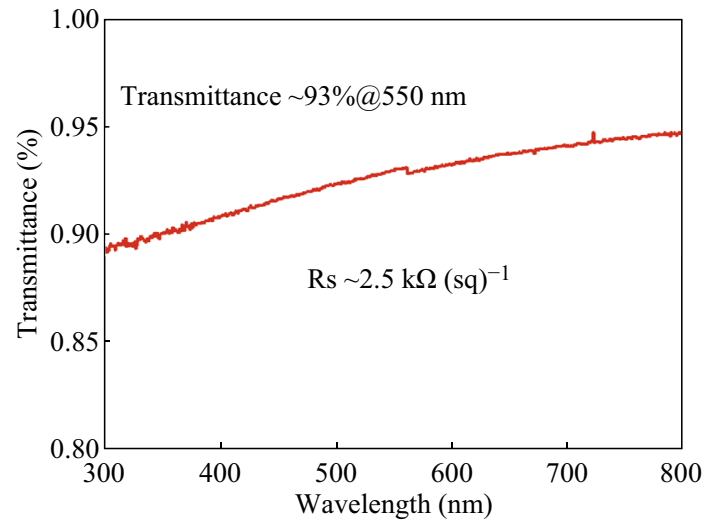

Fig. 5 The optical transmittance spectrum of the $\mathrm{F}_{16} \mathrm{CuPc}$-derived doped graphene on a quartz wafer. Inset is the sheet resistance measured by four-point probe measurement

$\sim 93 \%$ at $550 \mathrm{~nm}$, even though the doped graphene film is 3-4 layers. The sheet resistance $\left(R_{\mathrm{S}}\right)$ obtained from fourpoint probe measurement is $\sim 2.5 \mathrm{k} \Omega(\mathrm{sq})^{-1}$, revealing that the as-grown N, F-co-doped graphene film is of high conductivity.

\section{Conclusions}

In summary, a facile method for high-quality synthesis of doped graphene film on the dielectric substrate has been developed. PAHs containing dopant elements with planar configuration were used as both carbon feedstocks and doping sources and a layer of $\mathrm{Cu}$ film as the catalyst. The thickness of $\mathrm{Cu}$ layer and PAHs, the annealing time, and temperature are optimized for high-quality graphene growth. N-doped and N, F-co-doped graphene have been synthesized using TPB and $\mathrm{F}_{16} \mathrm{CuPc}$ as solid carbon sources, respectively. The properties of the as-grown samples were well studied and N, F-co-doped graphene exhibits a high optical transmittance and low sheet resistance. The present growth strategy provides a controllable transferfree route for high-quality doped graphene growth, which 
will facilitate the practical electronic applications of graphene.

Acknowledgments The work was supported by Natural Science Foundation of China (NSFC) (Grant No. 91333112, U1432249), the Priority Academic Program Development of Jiangsu Higher Education Institutions. This project has also been supported by Collaborative Innovation Center of Suzhou Nano Science \& Technology and sponsored by Qing Lan Project.

Open Access This article is distributed under the terms of the Creative Commons Attribution 4.0 International License (http://crea tivecommons.org/licenses/by/4.0/), which permits unrestricted use, distribution, and reproduction in any medium, provided you give appropriate credit to the original author(s) and the source, provide a link to the Creative Commons license, and indicate if changes were made.

\section{References}

1. C.G. Lee, X.D. Wei, J.W. Kysar, J. Hone, Measurement of the elastic properties and intrinsic strength of monolayer graphene. Science 321(18), 385-388 (2008). doi:10.1126/science.1157996

2. X. Du, I. Skachko, A. Barker, E.Y. Andrei, Approaching ballistic transport in suspended graphene. Nat. Nanotechnol. 3, 491-495 (2008). doi:10.1038/nnano.2008.199

3. K.S. Kim, Y. Zhao, H. Jang, S.Y. Lee, J.M. Kim, J.H. Ahn, P. Kim, J.Y. Choi, B.H. Hong, Large-scale pattern growth of graphene films for stretchable transparent electrodes. Nature 457, 706-710 (2009). doi:10.1038/nature07719

4. J.H. Seol, I. Jo, A.L. Moore, L. Lindsay, Z.H. Aitken et al., Twodimensional phonon transport in supported graphene. Science 328, 213-216 (2010). doi:10.1126/science. 1184014

5. X. Sun, L. Qiao, X. Wang, A novel immunosensor based on au nanoparticles and polyaniline/multiwall carbon nanotubes/chitosan nanocomposite film functionalized interface. Nano-Micro Lett. 5(3), 191-201 (2013). doi:10.5101/nml.v5i3

6. R.R. Nair, P. Blake, A.N. Grigorenko, K.S. Novoselov, T.J. Booth, T. Stauber, N.M.R. Peres, A.K. Geim, Fine structure constant defines visual transparency of graphene. Science 320, 1308 (2008). doi:10.1126/science. 1156965

7. A.K. Geim, Graphene: status and prospects. Science 324, 1530-1534 (2009). doi:10.1126/science.1158877

8. Yu. Lili, Wu Hui, Wu Beina, Ziyi Wang, Hongmei Cao, Fu Congying, Nengqin Jia, Magnetic $\mathrm{Fe}_{3} \mathrm{O}_{4}$-reduced graphene oxide nanocomposites-based electrochemical biosensing. Nano-Micro Lett. 6(3), 258-267 (2014). doi:10.5101/nml140028a

9. Y. Xue, B. Wu, L. Jiang, Y. Guo, L. Huang et al., Low temperature growth of highly nitrogen-doped single crystal graphene arrays by chemical vapor deposition. J. Am. Chem. Soc. 134(27), 11060-11063 (2012). doi:10.1021/ja302483t

10. W. Haixia, Q. Liu, S. Guo, Composites of graphene and $\mathrm{LiFePO}_{4}$ as cathode materials for lithium ion battery: a mini-review. Nano-
Micro Lett. 6(4), 316-326 (2014). doi:10.1007/s40820-014-00046

11. L.T. Qu, Y. Liu, J.B. Baek, L.M. Dai, Nitrogen-doped graphene as efficient metal-free electrocatalyst for oxygen reduction in fuel cells. ACS Nano 4(3), 1321-1326 (2010). doi:10.1021/ nn901850u

12. Z. Yang, Z. Yao, G.F. Li, G.Y. Fang, H.G. Nie, Z. Liu, X.M. Zhou, X.A. Chen, S.M. Huang, Sulfur-doped graphene as an efficient metal-free cathode catalyst for oxygen reduction. ACS Nano 6(1), 205-211 (2011). doi:10.1021/nn203393d

13. A.L.M. Reddy, A. Srivastava, S.R. Gowda, H. Gullapalli, M. Dubey, P.M. Ajayan, Synthesis of nitrogen-doped graphene films for lithium battery application. ACS Nano 4(11), 6337-6342 (2010). doi:10.1021/nn101926g

14. A. Reina, X.T. Jia, J. Ho, D. Nezich, H. Son, V. Bulovic, M.S Dresselhaus, J. Kong, Large area, few-layer graphene films on arbitrary substrates by chemical vapor deposition. Nano Lett. 9(1), 30-35 (2009). doi:10.1021/n1801827v

15. X.S. Lia, W.W. Caia, J. Ana, S. Kimb, J. Nahb et al., Large-area synthesis of high-quality and uniform graphene films on copper foils. Science 324, 1312-1314 (2009). doi:10.1126/science. 1171245

16. Z.Q. Luo, S.H. Lim, Z.Q. Tian, J.Z. Shang, L.F. Lai, B. MacDonald, C. Fu, Z.X. Shen, T. Yu, J.Y. Lin, Pyridinic N-doped graphene: synthesis, electronic structure, and electrocatalytic property. J. Mater. Chem. 21, 8038-8044 (2011). doi:10.1039/ c1jm10845j

17. Z. Jin, J. Yao, C. Kittrell, J.M. Tour, Large-scale growth and characterizations of nitrogen-doped monolayer graphene sheets. ACS Nano 5(5), 4112-4117 (2011). doi:10.1021/nn200766e

18. Z. Sun, Z. Yan, J. Yao, E. Beitler, Y. Zhu, J.M. Tour, Growth of graphene from solid carbon sources. Nature 468, 549-552 (2010). doi:10.1038/nature09579

19. L. Britnell, R.V. Gorbachev, R. Jalil, B.D. Belle, F. Schedin et al., Field-effect tunneling transistor based on vertical graphene heterostructures. Science 335, 947-950 (2012). doi:10.1126/sci ence. 1218461

20. S. Bae, H. Kim, Y. Lee, X.F. Xu, J.S. Park et al., Roll-to-roll production of 30-inch graphene films for transparent electrodes. Nat. Nanotechnol. 5(8), 574-578 (2010). doi:10.1038/nnano. 2010.132

21. Q. Zhuo, Q. Wang, Y.P. Zhang, D. Zhang, Q.L. Li et al., Transfer-free synthesis of doped and patterned graphene films. ACS Nano 9, 594-601 (2015). doi:10.1021/nn505913v

22. D. Graf, F. Molitor, K. Ensslin, C. Stampfer, A. Jungen, C. Hierold, L. Wirtz, Spatially resolved raman spectroscopy of single- and few-layer graphene. Nano Lett. 7(2), 238 (2007). doi:10.1021/n1061702a

23. A.C. Ferrari, J.C. Meyer, V. Scardaci, C. Casiraghi, M. Lazzeri et al., Raman spectrum of graphene and graphene layers. Phys. Rev. Lett. 97, 187401 (2006). doi:10.1103/PhysRevLett.97. 187401

24. R. Stine, W.K. Lee, K.E. Whitener Jr, J.T. Robinson, P.E. Sheehan, Chemical stability of graphene fluoride produced by exposure to $\mathrm{XeF}_{2}$. Nano Lett. 13, 4311-4316 (2013). doi:10. 1021/n14021039 